\title{
Scattered Light in H II Regions
}

\author{
C. R. O'DELL \\ Yerkes Observatory \\ Williams Bay, Wisconsin
}

The possible role of interstellar particles in the determination 1 of the ionization structure of $\mathrm{H}$ II regions, the transfer of the Lyman line radiation, and the early stages of stellar contraction have stimulated an investigation of the properties and relative abundance of the scattering particles that exist within the boundaries of a number of bright galactic diffuse nebulae. It is interesting to note that the conclusion reached in reference 1 , based on crude observations, was that there does not seem to be any scattered light in the bright $\mathrm{H}$ II regions; this conclusion is in contradiction to the results of the study presented herein. Results of the present investigation show that, in general, dust is an important constituent of the diffuse nebulae; that certain anomalies in the gas-to-dust ratio can be explained in a straightforward manner through the interaction of the particles and stellar radiation; and that the anomalies are closely related to the deviations known to exist in the interstellar reddening law.

The apparent surface brightnesses in the emission line $\mathrm{H} \beta$ and the strengths relative to $\mathrm{H} \beta$ of the continuous radiation at optical wavelengths have been investigated in 15 diffuse nebulae by using the methods of photoelectric filter photometry. Of these 15 nebulae, NGC 1976 (ref. 2), NGC 6514, NGC 6523, and NGC 6611 (ref. 3) have been investigated in detail across the apparent surface, enabling us to study the nature and amount of material inside the region of ionized gas. It was seen that almost all the nebulae investigated have a continuum that is stronger than that which is predicted from purely atomic processes; the excess is attributed to light scattered within the nebulae. Figure 1 gives the relevant data for the nebulae observed. The strength of the continuum can be given as an equivalent width relative to $\mathrm{H} \beta$ in emission and should be compared with the theoretical value of $1150 \AA$. This measure is in the sense that a smaller equivalent width denotes a stronger continuum and is not affected by the amount of interstellar extinction. The surface brightnesses given are not corrected for the effects of ex- 


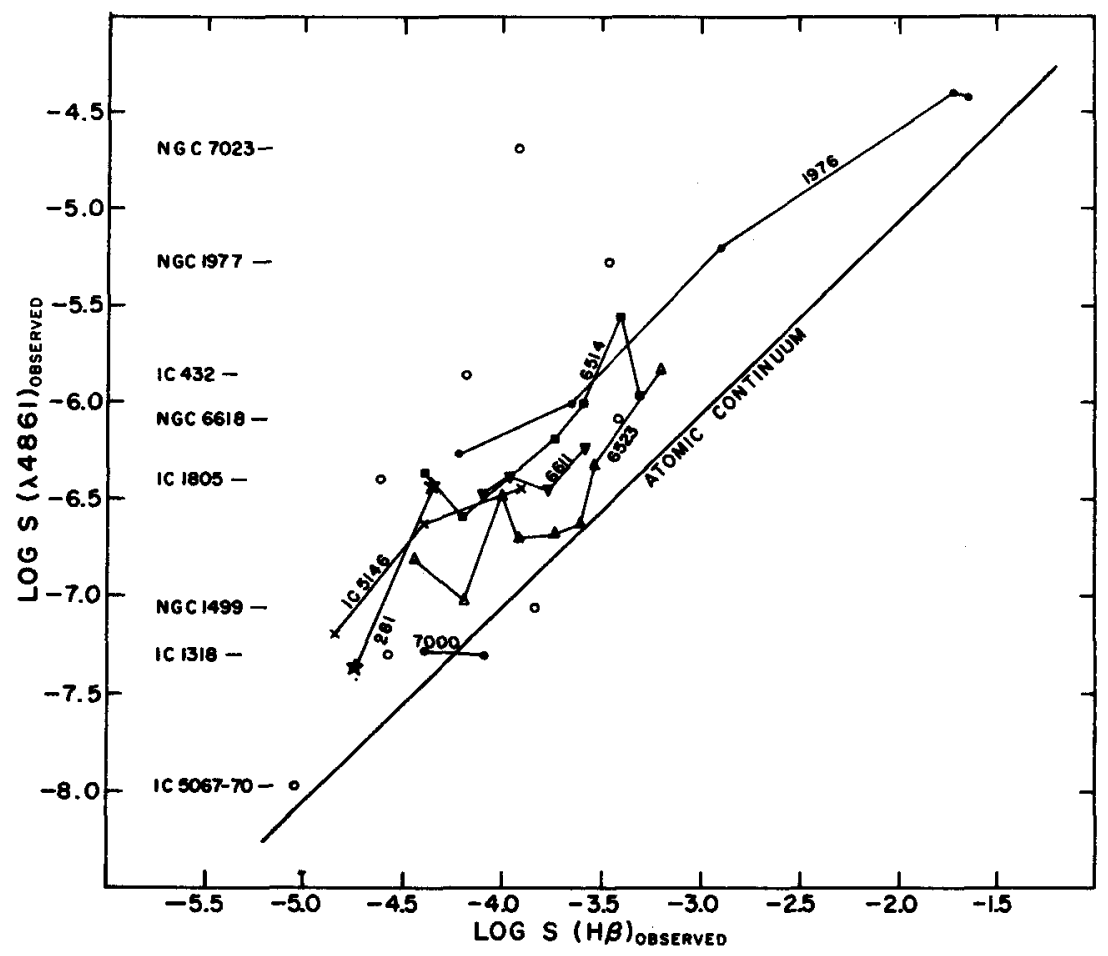

FIGURE 1. - Surface brightness of galactic nebulae in the continuum at $\lambda 4861\left(\mathrm{ergs} / \mathrm{cm}^{2} /\right.$ $\mathrm{sec} / \mathrm{steradian} / \AA$ ) as a function of surface brightness in the emission line $\mathrm{H} \beta$ (ergs $/ \mathrm{cm}^{2}$ ) sec/steradian). Observations of multiple points in the same nebula are connected by a thin solid line. The heavy line labeled "atomic continuum" shows the predicted relation for a purely gaseous nebula.

tinction. The multiple positions for individual nebulae refer to different distances from the exciting star (or stars). The equivalent widths are generally about 400 and range from $59 \AA$ to $1670 \AA$.

The effective gas-to-dust ratio can be determined from the following equation:

$$
N_{\mathrm{H}} / N_{d} \sigma_{\lambda}=N_{\mathrm{H}} / \int_{0}^{\infty} N(a) \pi a^{2} Q(a / \lambda) \mathrm{d} a
$$

where

$N_{\mathrm{H}} \quad$ density of ionized hydrogen

$N_{d} \quad$ density of dust

$\sigma_{\lambda} \quad$ extinction cross section for a dust particle

$N(a)$ size distribution of particles of radius $a$

$Q(a / \lambda)$ scattering efficiency at wavelength of the continuum measures 
The detailed investigations show that the effective gas-to-dust ratio is very similar in both the nebulae NGC 6523 and NGC 6611 and in the general interstellar medium, attaining a value of $N_{\mathrm{H}} / N_{d} \sigma_{\lambda}=20 \times 10^{20} /$ $\mathrm{cm}^{2}$. The Trifid Nebula (NGC 6514) 'seems to be particularly dusty, with $N_{\mathrm{H}} / N_{d} \sigma_{\lambda}=4 \times 10^{20} / \mathrm{cm}^{2}$. The most interesting object is NGC 1976, where there is a wide variation in the effective gas-to-dust ratio from 144 $\times 10^{20} / \mathrm{cm}^{2}$ in the inner regions to $5 \times 10^{20} / \mathrm{cm}^{2}$ in the outer regions of the nebula. This variation occurs because there is a smaller effective scattering cross section per ionized hydrogen atom in the inner regions of the nebula. It is significant that the most deviant reddening curve known is also found in the inner region of the Orion Nebula (ref. 4). a fact utilized in the interpretation of these observations.

This new material is evaluated under rather simplified considerations of the interaction of the grain and the radiation inside the nebulae. There seem to be two main processes that can produce the observed characteristics; grain evaporation and grain repulsion by radiation pressure. In determining the rate of evaporation, we have considered the input of energy through absorption of both the stellar radiation and the Lyman line radiation and through electron impact. The energy input by these mechanisms is counteracted by infrared thermal emission by the grains and is used to find the rate at which the particles are heated above the temperature required for evaporation to occur. The procedure adopted in reference 5, where evaporation is assumed to occur when the vapor pressure exceeds the stoichiometric pressure of the particles, is followed to evaluate the grain temperatures necessary to evaporate grains composed mostly of $\mathrm{CH}_{4}, \mathrm{NH}_{3}$, and $\mathrm{H}_{2} \mathrm{O}$; temperatures of $28^{\circ} \mathrm{K}$, $75^{\circ} \mathrm{K}$, and $105^{\circ} \mathrm{K}$, respectively, were found for the low densities of the interstellar medium critical grain. The two principal energy-input mechanisms are through absorption of the stellar radiation and absorption of resonance line radiation; the energy input by complete transfer of energy from free electrons is small compared with these processes. The absorption efficiency of particles with radii about the wavelength of visual radiation is quite high since most of the radiation is coming out at much shorter wavelengths. The energy input by the stellar continuum is very sensitive to the effective temperature and radius of the exciting star and is strongly concentrated to the central nebular regions because of the inverse square dilution factor. On the other hand, the energy input due to absorption of Lyman line radiation is fairly homogeneous because of the nearly constant Lyman $\alpha$ flux density. Except for the innermost regions, the energy input by absorption of Lyman $\alpha$ radiation dominates by a factor of about 2 . Since the radiating efficiency decreases for decreasing grain size, the smaller grains are at a higher equilibrium temperature. If all grains smaller than a critical size $a_{e}$ have a temperature higher than that necessary for evaporation, then very 
soon all such grains would disappear, while the larger, cooler grains would not be modified significantly. For the Orion nebula the critical size would be $a_{e}(\mu)=0.03 / \phi^{2}$ for $\mathrm{H}_{2} \mathrm{O}, a_{e}(\mu)=0.17 / \phi^{2}$ for $\mathrm{NH}_{3}$ grains, and $a_{e}=22.7 / \phi^{2}$ for $\mathrm{CH}_{4}$ grains, where $\phi$ is the apparent displacement from the Trapezium cluster in arc minutes. Since the most effective size in scattering photons of wavelength $4861 \AA$ is about $0.2 \mu$, grain evaporation may be considered unimportant in this nebula unless the grains are destroyed when $\mathrm{CH}_{4}$ is evaporated, a result not consistent with the observations of grains in other $H$ II regions. Since this is the most favorable case, the conclusion is very likely generally valid.

The effects of radiation pressure expulsion of grains promise to be far more important in most $H$ II regions. This effect, selectively being stronger for the smaller particles, causes grains to be pushed out of the vicinity of the most luminous stars. One would expect the grains to be accelerated to a velocity approaching that where the drag caused by collisions with the gas particles equals the force of radiation pressure. Since the drag increases as the square of the relative velocity, the particles will rapidly accelerate until they reach a velocity close to the terminal velocity and then proceed at nearly the terminal velocity - which depends upon the gas density and the distance from the central star (or stars). If the age of the Orion Nebula is $10^{5}$ to $10^{6}$ years, then one can explain the observed variation in the effective gas-to-dust ratio on the basis of radiation pressure expulsion.

This interpretation of the scattered light observations is strengthened from the known peculiarities of the reddening law for the stars in and close to the Trapezium. The selective acceleration of the smaller particles would mean that they have been pushed out of the most dense region, preferentially leaving the larger grains behind where most of the reddening occurs. If the reddening law for Orion before the illumination occurred were similar to that found in the Cygnus region of the Galaxy, then this selective depletion of small particles would cause the original reddening curve to be modified into the flat curve that is now observed. This same mechanism must also be operating in the other diffuse nebulae; but, in the case of the other nebulae investigated in detail, one can show that the most luminous stars have shown for an insufficient time to cause drastic changes in the amount of dust. However, the stars have been bright long enough to have caused some small changes in the dust, a fact consistent with Johnson's observations that the reddening curve is slightly modified in these regions.

The fact that the amount of dust in the outer regions of NGC 1976 and in NGC 6514 is greater than that in the general interstellar medium may be interpreted as evidence for accelerated grain growth in regions of higher density. It would appear that gradual accretion by sticking collisions is very important in the growth of grain nuclei, a process which 
depends upon the mean temperature and density of the region in which the grains are found. Since it is very likely that grains existed in the original clouds which now constitute these larger $\mathrm{H}$ II regions, the rate of growth should have been accelerated. For grains composed of ices, in a cloud of temperature $100^{\circ} \mathrm{K}$ and having final radii of $0.2 \mu$, the grain growth time is about $6 \times 10^{8} / N_{\mathrm{H}}$ years. For the Trifid Nebula this timescale becomes about $10^{7}$ years, sufficiently short that we are now probably observing regions where the grains have recently grown rapidly in size but where the illuminating stars have not had time to push out many of the smaller grains. If grain growth is accelerated in the neutral clouds of material, then what we see in the other H II regions may be the combination of recent grain growth and radiative expulsion. It is interesting to note that a substantial fraction of the common heavy elements are tied up in the grains if they are icelike. For the values of the cosmic abundance usually assumed, the limiting value would be $N_{\mathrm{H}} / N_{d} \sigma_{\lambda}$ $=3 \times 10^{20} / \mathrm{cm}^{2}$ if the effective grain radius is $1000 \AA$ and all $\mathrm{O}, \mathrm{C}$, and $\mathrm{N}$ are in grains. This value is very close to the values observed in the outer Orion Nebula and the Trifid Nebula. Possibly in these regions the accretion has gone close to completion, a hypothesis that could be tested by accurately determining the relative atomic abundances.

The question that remains is: How important are these grains in the study of the $\mathrm{H}$ II regions; specifically, what are their roles in the determination of the ionization structure, the transfer of resonance radiation, and the evolution of the nebulae? If the effective gas-to-dust ratio is $20 \times 10^{20} / \mathrm{cm}^{2}$ (probably an overestimate due to recent grain growth) at $\lambda=4861 \AA$, the total optical depth at $\mathrm{H} \beta$ for an $\mathrm{H}$ II region illuminated by a single $\mathrm{O} 5$ star will be 1.0 . Since the radiation causing the ionization of hydrogen falls at a much shorter wavelength, the ultraviolet optical depth will be considerably larger - an effect counteracted in part by the fact that only the true absorption component will be important. This means that significant fractions of the energy from the star, that otherwise would go toward ionization of neutral hydrogen, will be lost by grain absorption and reradiation into the infrared. This would cause the degree of ionization to decrease more rapidly in the inner regions and make the total Strömgren sphere smaller. The problem for the transfer of Lyman $\alpha$ radiation is even more extreme because the photons are scattered by the absorption-emission process of hydrogen. This scattering causes the average Lyman $\alpha$ photon to traverse a total path inside the nebula that is larger than the radius by a factor of at least 10 , and possibly much larger because of the effects of back scattering by the outer neutral shell. (See refs. 6 and 7.) The probability that a Lyman $\alpha$ photon will be destroyed by absorption by a grain is, therefore, almost certain. This fact can also be true for other optically thick Lyman line photons and can cause a significant deviation from the elementary conditions of statistical 
equilibrium that are assumed in the calculation of the emissivities in Balmer radiation. Finally, the continued rapid grain growth in the outer, un-ionized regions of an interstellar cloud can modify the nature of the structure and evolution of the H II region because of the expected sharp discontinuity in the relative opacity in the continuum. In this instance, the ionization front would not grow as rapidly as expected in a grainfree nebula, and the shock front concentration of material (bulldozing) ahead of the ionization boundary would be of greater importance and relatively thicker than previously calculated for a pure gas nebula.

The present results have shown that interstellar grains do exist in abundance in the ionized regions of the interstellar medium; mechanisms have been outlined that may be important in understanding the nature of the particles. The need to extend this work to regions of the galaxy known to have anomalous scattering properties is obvious. It would appear that, to a first approximation, Hubble's division of nebulae into emission-line and reflection objects is because of the nature of the ionization field and does not constitute an intrinsic difference as suggested in reference 1 .

\section{REFERENCES}

1. Shajn, G. A.; Haze, V. T.; and Pikelner, S. B.: Proceedings of the Liège Symposia, vol. 15,1954, p. 441.

2. O'Dell, C. R.; and HubBard, W. B.: Photoelectric Spectrophotometry of Gaseous Nebulae I. The Orion Nebula. Astrophys. J., vol. 142, 1965, p. 591.

3. O’Dell, C. R.; Hubbard, W. B.; and Peimbert, M.: Photoelectric Spectrophotom. etry of Gaseous Nebulae III. Scattered Light in Three Bright H II Regions. Astrophys. J., vol. 143, 1966, p. 743 .

4. Johnson, H. L.: Interstellar Extinction in the Galaxy. Astrophys. J., vol. 141, 1965, p. 923.

5. Gaustad, J. E.: The Opacity of Diffuse Cosmic Matter and the Early Stages of Star Formation. Astrophys. J., vol. 138, 1963, p. 1050.

6. O'DeLL, C. R.: Photoelectric Spectrophotometry of Gaseous Nebulae II. The Reflection Nebula Around Merope. Astrophys. J., vol. 142, 1965, p. 604.

7. Yada, B.; and Osakı, T.: The Radiation Field in a Galactic Nebula with an H I Envelope I. Astron. Soc. Japan, Pub., vol. 9, 1957, p. 82.

\section{DISCUSSION}

Walker: How do you compare the observed continuum emission with that to be expected from free-free emission? Did you actually look at any planetary nebulae?

O'Dell: This was done before. The level of the emission there is generally that found, within the observational errors.

Walker: The values should fall on the theoretical straight line in your diagram, of course. I think that would be very crucial for your argument. 
O'Dell: Yes. In the case of some of these very strong continuum objects for which the continuum is 10 times too strong, the argument is very safe; however, in the case of NGC 7000, for which the continuum accords with the predicted amount using the excess $\mathrm{H} \beta$ to define light scattered within the nebula becomes very dangerous. That is why I haven't tried to interpret most of these nebulae but have limited myself to the ones that did have a strong continuum. They have a strong continuum because of the low dilution factors, and accurate values can be derived for these parameters. The other 11 nebulae were not discussed in detail because they do not have strong continua, and because I did not get enough observations to make any valid discussion.

Donn: Is there any evidence that the CNO lines in the outer regions of the Orion nebula are weaker in the spectrum than in the center? Another point related to this is that the study of the radial volocity indicated a high degree of turbulence in the Orion nebula. What about this material over extensive regions of the nebula? Would this be an important factor in your argument?

O'Dell: It would if the turbulence had a scale the size of the nebula. But the turbulence rather has a scale the size of seconds of arc; that is, 10 to 20 seconds of arc rather than 5 to 10 minutes of arc. In answer to your first question, I don't know about the CNO lines.

Wickramasinghe: I would like to point out that you have to be a little careful in the radiation pressure arguments. Very small particles would not necessarily be pushed out. It depends upon the index of refraction.

O'Dell: This I omitted because of a lack of time. We have investigated the Mie theory scattering for particles. What we've done is to try to fit the Cygnus law by using the wavelength dependence of the index of refraction in the most accurate manner possible with programing, and we used only the wavelength-dependent $Q$ that is relevant to radiation pressure.

Greenberg: Certainly at $30000^{\circ} \mathrm{K}$ to $50000^{\circ} \mathrm{K}$, for example, the as the ultraviolet (these are the dominant ones in these cases I believe), we have found that the small particles are pushed out more than the big ones as long as the stellar temperatures are high.

O'Dell: What is high and low?

Greenberg: Certainly at $30000^{\circ} \mathrm{K}$ to $50000^{\circ} \mathrm{K}$, for example, the small grains will be pushed out.

O'Dell: But that is what we are talking about in the H II regions.

Voice: Are you thinking of ices?

Greenberg: Yes. I have a question: what size scale do you use to say whether or not the radiation pressure is effective in the cases you have given here? For example, what is the size scale that you have used for the inner portion of Orion? 
O'Dell: The radius, the apparent radius.

Greenberg: What is that in terms of parsecs? You need a time scale and a distance scale.

O'Dell: I derived the distance for each of these nebulae independently, except for Orion where I adopted 430 parsecs.

Greenberg: What did you call the inside of Orion?

O'Dell: I called that the innermost 4 arc minutes.

Greenberg: How much is that in parsecs?

O'Dell: That is $1 / 2$ parsec, or more exactly $4 / 7$ of a parsec. Orion is special because it has a much lower dilution factor, that is, the material is much more concentrated than is typical for H II regions.

Nandy: What bearing does your finding have on the rate of star formation? This is a very good related subject.

O'Dell: I am not trying to speculate in these directions, except for the fact that if star formation is accelerated by an increase in the uptical depth in the continuum produced by grain growth, then perhaps NGC 6514 and the outer parts of Orion show that the grain growth can occur on a short time scale and that dust would tend to accelerate the mechanism.

Nandy: Could we say that the rate of star formation would be different in different regions?

O'Dell: You could say that.

Wickramasinghe: I think some theoretical work has been done by W. Fowler and E. Hoyle on star formation and its relation to grain opacity. They came to the conclusion that the opacity of the grains played a very crucial role in determining whether or not you get certain types of contraction processes. Also, they concluded that the nature of the dust plays an important role in determining the luminosity function.

O'Dell: Thank you. All that I can say is that the dust would probably accelerate stellar contraction.

Hall: Will you say how far out the outer regions are that you are talking about?

O'Dell: In Orion? I think 18.3 arc minutes is generally considered the outermost region.

Hall: Are there any polarization measurements?

O'Dell: No. The regions of overlap, actually my regions two and three, were the regions that had a very strong continuum.

Field: Concerning your calculations of the atomic continuum to which, I believe, Dr. Walker referred: I am not sure I understand your answer to that. Did you account for the 2-photon emission?

O'Dell: Yes. Essentially the atomic continuum that I considered was the free-bound, the free-free, and the 2-photon, from $2 \mathrm{~s}$ to $1 \mathrm{~s}$, emission. 
Field: Doesn't the 2-photon emission depend upon the degree of Lyman $\alpha$ trapping, because Lyman $\alpha$ can populate the $2 \mathrm{~s}$ level via $2 s-2 p$ collisional transitions?

O'Dell: Only in the case of extremely large optical depths for Ly$\operatorname{man} \alpha$.

Field: That's true.

O'Dell: I would rather assume that the grains never let the penetration depth get this large. In other words the Lyman alpha radiation would be destroyed before $10^{6}$ scatterings would occur.

Field: Yes, but unfortunately you can't use the presence of the grains in this argument, because, if indeed the continuum is due to the 2 -photon process, there would be no grains at all.

O'Dell: The color of the continuum is not consistent in low density regions. Sometimes the extra continuum is red while the 2-photon component is extremely blue.

Field: Another question concerns the general calculation of radiation pressure. I didn't understand whether in fact the grains did come to a terminal velocity or not in the times available.

O'Dell: All have achieved a terminal velocity.

Field: I see. If that is true, and they have reached terminal velocity, then I think your statement that the small grains would be pushed out faster may not be correct.

O'Dell: That is right, if you let the process of grain ejection run long enough.

Field: If you let it run long enough, the terminal velocity will be that of the larger grains because the extinction efficiency is greater. The point is that the drag force is proportional to the area and the radiation pressure is proportional to the area times the efficiency. For the small grains the efficiency would be small; therefore, the velocity of the small grains would be smaller than that of the large ones.

O'Dell: That is certainly true.

Field: I have one more question. In your calculation of the heating of the grains you didn't go into details. Did you account for the fact that beyond the dissociation limit of water the absorptivity of the particle is going to go up very drastically and therefore, presumably, the particle would heat up more effectively? What did you assume about the absorptivity?

O'Dell: I assumed that it was quite high, essentially 1 . I used the value obtained from simple Mie scattering, the Mie absorption in this case. I didn't consider the effects of bands here.

Donn: You now have this grain in a high energy medium with a temperature of the order of a few thousand degrees in the Orion nebulae. The density that you get is on the order of 1000 or 10000 
electrons $/ \mathrm{cm}^{3}$. Now what contribution does this provide to the energy of the particle?

O'Dell: I computed the energy input assuming that for every electron collision the electron gave up the total amount of its kinetic energy. This is small compared with the other two mechanisms of energy input.

Donn: What about particle collisions?

Greenberg: They are negligible. 